\title{
Temperature-Dependent Structural Evolution of Pt-Ni Nanoparticles Observed by In Situ TEM
}

\author{
Xiner Lu and Kai He \\ Clemson University, Clemson, South Carolina, United States
}

The potential availability of renewable energy is highly dependent on the production of high-performance electrocatalysts for energy conversion and storage applications. In the past years, there has been an increasing interest in synthesizing Pt alloy nanocrystals to achieve high catalytic performance for electrolyzers and fuel cells. Pt-Ni nanoparticles (NPs) have shown high electrocatalytic activity for the oxygen reduction reaction at the cathode [1]. However, the stability of surface structures and compositions still needs to be improved to obtain better long-term catalytic stability. It is noted that thermal annealing can adjust the surface structures and compositions to improve the stability. Transmission electron microscopy (TEM) plays a vital role in characterizing the morphology, structure, and composition of NPs. Specifically, the utilization of in situ TEM can allow for observing real-time evolution of materials transformation during dynamic processes or reactions. With the help of microelectromechanical system (MEMS) technique, special in situ TEM platforms can be fabricated to enable a variety of functions, such as heating, biasing, gas cell, liquid cell, and so on [2].

The solution phase synthesis is proven to be an effective method for the control of NPs with monodisperse size distribution and well-defined shapes [3]. We have produced single-crystalline Pt-Ni NPs in truncated octahedral shape with $\{111\}$ terminating facets using thermal decomposition synthesis, as demonstrated by high-resolution TEM (HRTEM) and X-ray diffraction (XRD) in Figure 1. For further improvement of the cost effectiveness and catalytic performance, we intend to add extra amount of Ni precursor to produce Ni-rich PtNi NPs. However, it is still poorly understood how the resulting structural and compositional inhomogeneity would evolve with respect to the change of temperature. Here, we have performed in situ heating experiments on Pt-Ni NPs at different temperatures to study their structural evolution under thermal annealing. Figure 2 shows a series of time-resolved TEM images of Pt-Ni NPs under in situ heating conditions with the temperature continuously rising from $30^{\circ} \mathrm{C}$ to $800^{\circ} \mathrm{C}$. We found that the NPs did not show any obvious change during thermal annealing below $300^{\circ} \mathrm{C}$, while the sharp vertices of the octahedra became rounded between $400^{\circ} \mathrm{C}$ and $500^{\circ} \mathrm{C}$ and completely turned into the spherical shape when heated above $600^{\circ} \mathrm{C}$. In addition, we also noted that some neighboring particles (marked by red circles) could coalesce to into one particle at $550^{\circ} \mathrm{C}-600^{\circ} \mathrm{C}$, which further became rounded at $700^{\circ} \mathrm{C}-800^{\circ} \mathrm{C}$. Based on the real-time observation, we proposed atomic models to illustrate possible evolution mechanisms for coalescence and reconstruction, as shown in Figure $2 \mathrm{~b}$ and c. The size of NPs was gradually decreased with the increase of temperature in the entire field of view, which is attributed to the minimization of the total surface energy that requires the faceted nanocrystals to change their shapes from octahedron to sphere. Furthermore, the residual organic ligands and amorphous layers on the surface of NPs might burn out during thermal annealing, which could also lead to the decrease in size. In summary, our in situ TEM observations show the time-sequential structural evolution of Pt-Ni NPs during thermal annealing processes. Further investigation to link the structural evolution to the compositional change will provide better understanding of surface reconstruction mechanism to support the controllable synthesis and processing for reaching the balance of catalytic activity and stability in the bimetallic system. Our study has leveraged the powerfulness of in situ TEM to reveal the impact of temperature-dependent thermal treatment on the structural modification of nanocrystals and show implications for the design of catalytically effective nanocrystal geometry of Pt-Ni bimetallic catalysts [4]. 

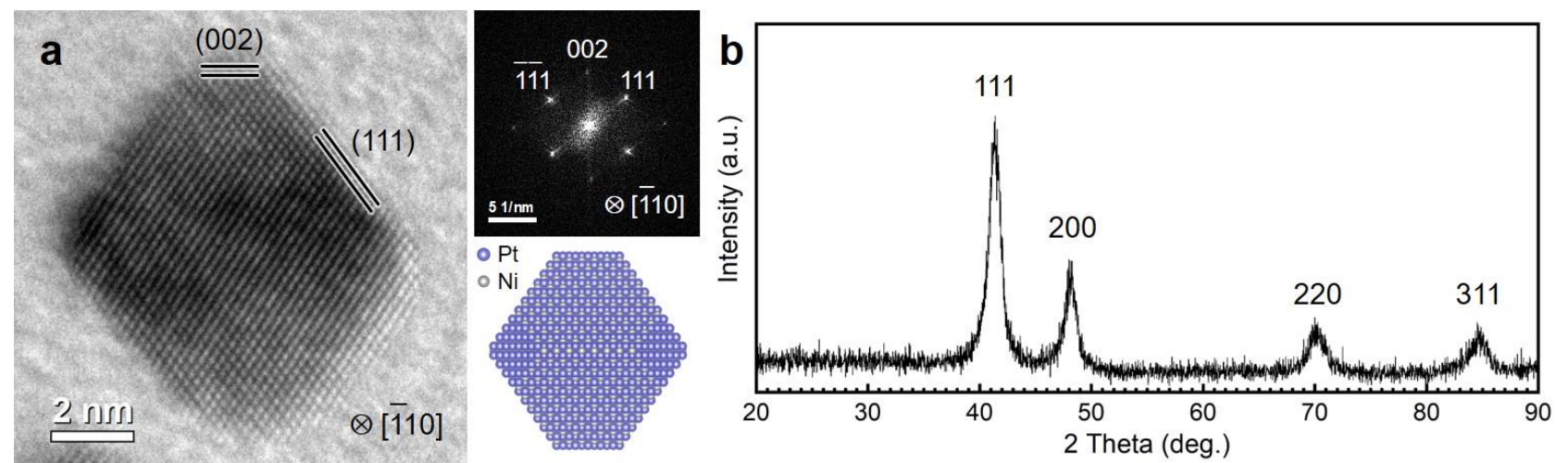

Figure 1. Figure 1. (a) HRTEM image with FFT and atomic model showing an individual Pt-Ni NP in truncated octahedral shape. (b) XRD pattern of as-synthesized Pt-Ni NPs.

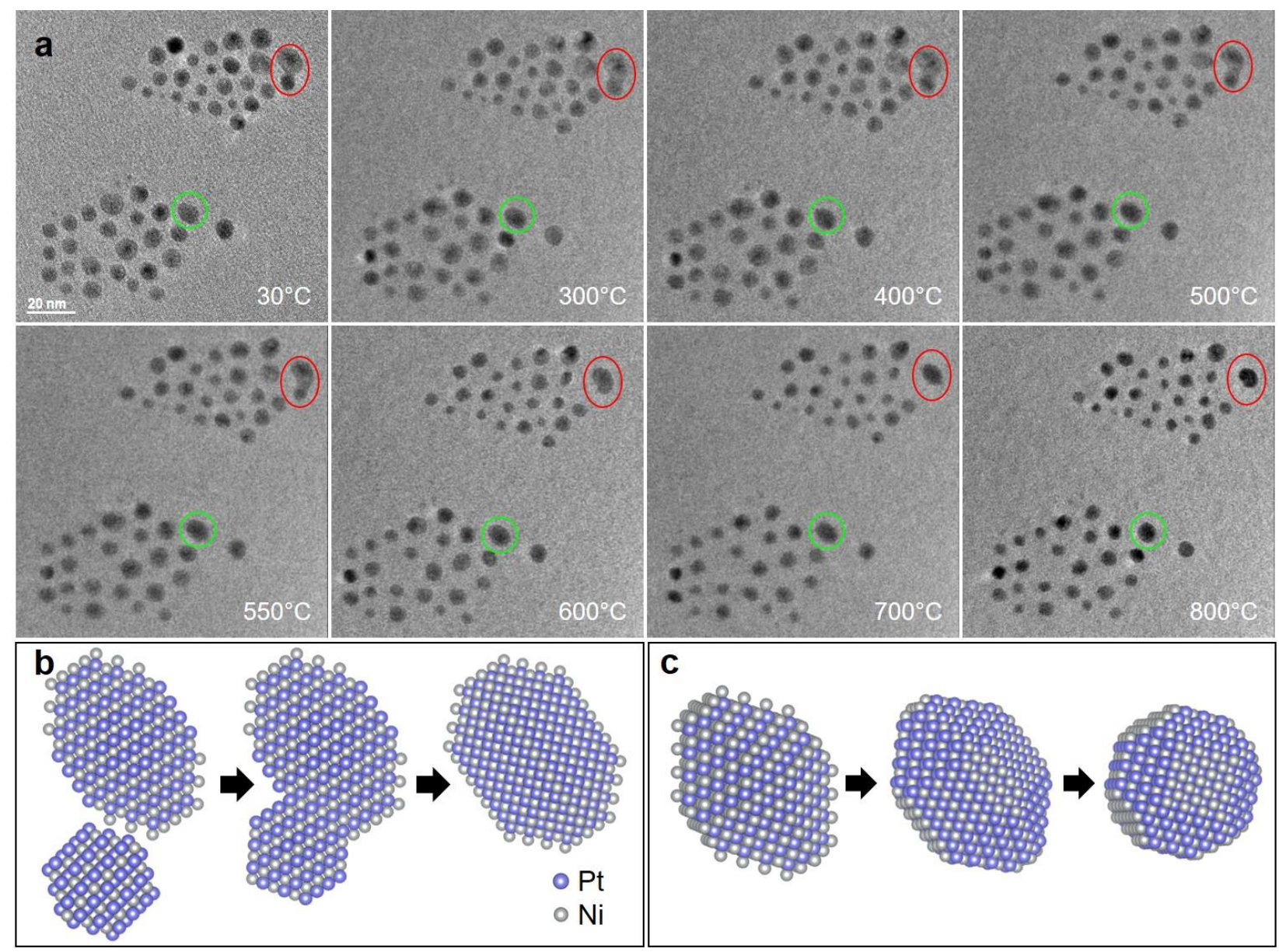

Figure 2. Figure 2. (a) TEM images showing the evolution of Pt-Ni NPs under in situ heating from $30^{\circ} \mathrm{C}$ to $800^{\circ} \mathrm{C}$. Atomic models of (b) coalescence and (c) reconstruction mechanisms, corresponding to the NPs marked by the red and green circles.

\section{References}

[1] S. Choi et al., Nano Lett. 13, 3420-3425 (2013).

[2] J. Cui et al., Adv. Mater. 33, 2000699 (2021).

[3] Q. Chang et al., Nano Lett. 17, 3926-3931 (2017).

[4] The authors acknowledge the use of Electron Microscopy Facility at Clemson University. 